\title{
Low ADAMTS 13 plasma levels are predictors of mortality in COVID-19 patients
}

\author{
Mario Bazzan ${ }^{1} \cdot$ Barbara Montaruli $^{2} \cdot$ Savino Sciascia ${ }^{3}$ (D) Domenico Cosseddu ${ }^{2} \cdot$ Claudio Norbiato $^{4}$. \\ Dario Roccatello ${ }^{3}$
}

Received: 26 May 2020 / Accepted: 30 May 2020 / Published online: 18 June 2020

(c) Società Italiana di Medicina Interna (SIMI) 2020

\section{Dear Editor,}

Both arterial and venous thrombotic complications have been reported in patients affected by COVID-19, especially in those critically ill. An emerging body of evidence is supporting our understanding of a so-called Coronavirusassociated coagulopathy (CAC) [1]. From a clinical point of view, in the initial phase of the pandemic, numerous empirical therapeutic strategies have been used, to include the use of heparins (alone or in association), hydroxychloroquine, steroids, immunomodulators, as well as plasma from convalescent patients from COVID-19 [2, 3]. However, data are still limited as these approaches have been reported most of the time in non-controlled studies. Nevertheless, regardless the intrinsic limitations of the available studies, some promising results in improving the clinical outcome, to include the thrombotic complications, have been reported. From a laboratory perspective, few considerations are similarly needed. While aPTT is often prolonged in these patients, it does not seem to be associated with a bleeding tendency but rather to the presence of antiphospholipid antibodies, mainly the lupus anticoagulant [4, 5]. Similarly, albeit modest and inconstant thrombocytopenia, increased (sometimes dramatically high) levels of D-dimer, and slightly prolonged

Savino Sciascia

savino.sciascia@unito.it

1 Thrombosis and Haemostasis, CMID-Coordinating Center of Piemonte and Valle d'Aosta Network for Rare Diseases, Turin, Italy

2 Department of Laboratory Medicine, AO Ordine Mauriziano, Turin, Italy

3 CMID-Coordinating Center of Piemonte and Valle d'Aosta Network for Rare Diseases and Nephrology and Dialysis, Department of Clinical and Biological Sciences, S. Giovanni Bosco Hospital and University of Turin, Piazza del Donatore di Sangue 3, 10154 Turin, Italy

4 Internal Medicine, AO Ordine Mauriziano, Turin, Italy prothrombin time (PT) are commonly observed, the mechanisms supporting the developed of CAC are still elusive [1]. The International Society of Thrombosis and Haemostasis (ISTH) recently published an "interim guidance" aiming to define some characteristics of the CAC [6]. Among others, the ISTH interim guidance highlighted some frequently observed hemostatic alterations in patients with $\mathrm{CAC}$, to include D-dimer usually increased, prothrombin time usually only marginally deranged and platelets count usually within rage or slightly reduced. Those observation are in line with the preliminary observations at the beginning of the pandemic outbreak [6-8]. Critically, the mentioned hemostatic alternations differ from those usually observed during the disseminated intravascular coagulopathy $[9,10]$. While discriminating between a consumption coagulopathy vs. a thrombotic microangiopathy might be challenging, the differentiation is crucial for therapeutic purpose.

In order to further investigate the CAC, we tested ADAMTS-13 and von Willebrand factor (vWF) plasma levels in 88 consecutive PCR-proven COVID-19 admitted patients (main characteristics detailed in Table 1). Adamts 13 activity and von Willebrand antigen (vWF:Ag) measurements were performed using CliA activity assays (HemosIL Acustar ADAMTS13 activity, IL, Lexington, MA, USA).

ADAMTS-13 levels were significantly reduced in all COVID-19 patients (CP) when compared to healthy controls (HC) (CP, mean 48.71 $\pm 18.7 \%$, HC, $108 \pm 9.1 \%$; normal value 60-130\%). These deranged values are similar to those observed in patients with thrombotic thrombocytopenic purpura (TTP), while ADMTS 13 is not generally reduced during a DIC.

Antibodies direct to ADAMTS 13 (assessed by Bethesda assay) were tested in the 25 patients with lowest ADAMTS 13 levels. Both ADAMTS-13 activity and antibodies anti- ADAMTS-13 were tested on the same sample. No patients were found to have significantly increased levels of 
Table 1 Laboratory profile at hospital admission dividing patients according to the outcome

\begin{tabular}{llllll}
\hline & \multicolumn{2}{l}{ Not survivors $=9$} & \multicolumn{2}{l}{ Survivors $=79$} & $p$ \\
\hline Male $(N, \%)$ & 6 & $66.7 \%$ & 54 & $68.4 \%$ & 0.91 \\
Age (years: mean; SD) & 71.89 & 7.1 & 59.37 & 12.7 & 0.037 \\
PLTs (N/ $\mu$ L; mean \pm SD) & 139.65 & 81.4 & 267.9 & 163.09 & 0.022 \\
vWF (IU/dL, mean; SD) & 395.52 & 113.21 & 295.5 & 132.76 & 0.033 \\
ADAMTS-13 (\%, mean; SD) & 32.17 & 15.56 & 50.6 & 18.17 & 0.035 \\
PT/INR (mean; SD) & 1.22 & 0.30 & 1.1 & 0.11 & 0.055 \\
D-Dimer (ng/ml; mean \pm SD) & 4198.33 & 4947.33 & 1494.6 & 2024.17 & 0.106 \\
D-Dimer > 3000 ng/ml $(N, \%)$ & 5 & $55.6 \%$ & 7 & $(8.8 \%)$ & 0.0001 \\
F/U days (mean; SD) & 11.6 & 7.7 & 10.7 & 6.95 & 0.077 \\
\hline
\end{tabular}

$v W F$ von Willebrand factor, $P L T s$ platelets, $F / U$ follow-up

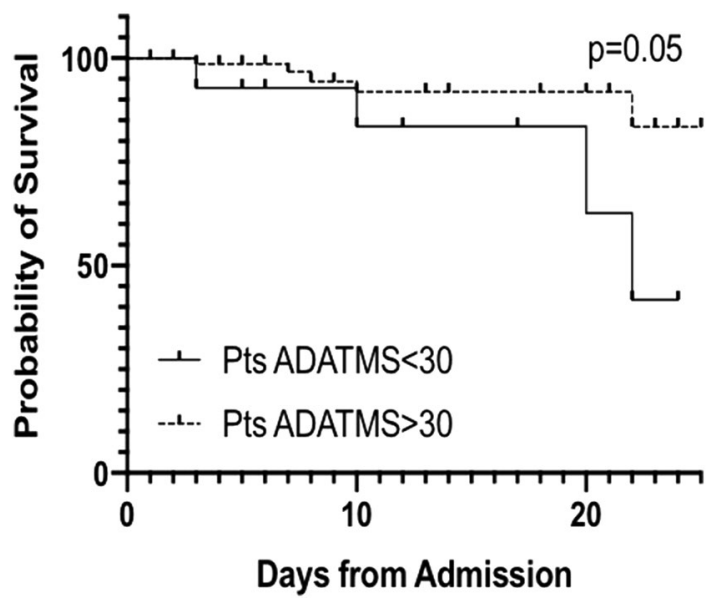

Fig. 1 Kaplan-Meier survival curves in COVID-19 patients according to ADATMS-13 plasma activity

anti- ADAMTS-13 antibodies (a borderline level was found in $1 / 25$ patient).

Overall, in our cohort we observed a mortality rate of $10.2 \%$ (9/88). Patients who died had significant lower levels of ADAMTS-13 and higher levels of von Willebrand factor (vWF) when compared to patients with non-fatal outcome (Table 1). After survival analysis, ADAMTS-13 plasma levels $<30 \%$ were significantly associated with a higher mortality (Fig. 1). Interestingly, as previously reported, also elevated levels of D-Dimer were associated with a fatal outcome $[4,5]$. Taken the above together, CAC features seems more in line with a thrombotic, TTP-like microangiopathy (almost normal hemostasis, elevated vWF high and low ADAMTS-13, platelet count slightly reduced) rather than with a DIC (PT and antithrombin levels reduced, reduced fibrinogen, PLTS variably reduced, ADAMTs-13 and vWF not described). These features could be related to the ADAMTS-13 "consumption" due to excess of circulating vWF (thrombotic tendency secondary to a "gain of factor"); the presence of anti-ADAMTS-13 antibodies was excluded in our cohort. In conclusion, high VWF plasma levels associated to low ADAMTS 13 could explain, at least in part, the strong thrombotic tendency in these patients. Our data could have the potential to guide possible new therapeutic options.

Acknowledgement The Authors are grateful to Bertero Maria Tiziana and Carignola Renato for their critical insight and to Bacco Beatrice and Gallo Cassarino Silvia for helping in the data collection.

Author contributions $\mathrm{MB}, \mathrm{SS}, \mathrm{BM}, \mathrm{DR}, \mathrm{CN}, \mathrm{DC}$ designed the study. SS and MB drafted the manuscript. BM performed laboratory testing. $\mathrm{DR}, \mathrm{CN}$ participated in data collection and analysis and critical interpretation of the results. All and reviewed the manuscript and approved the final version.

Funding This study was not supported by any specific grants/sponsor.

Data availability Available upon request.

\section{Compliance with ethical standards}

Conflict of interest The authors declare that they have no conflict of interest.

Ethics approval Ethical approval was obtained for this study by the local IRB.

Informed consent Consent was obtained from individuals included in this study in line with the protocol approved by the local IRB.

\section{References}

1. Tang N, Li D, Wang X, Sun Z (2020) Abnormal coagulation parameters are associated with poor prognosis in patients with novel coronavirus pneumonia. J Thromb Haemost. https://doi. org/10.1111/jth.14768

2. Sciascia S, Schreiber K, Radin M, Roccatello D, Krämer BK, Stach K (2020) Hydroxychloroquine reduces IL-6 and pro-thrombotic status. Autoimmun Rev 102555. https://doi.org/10.1016/j. autrev.2020.102555[Epub ahead of print]

3. Sciascia S, Aprà F, Baffa A et al (2020) Pilot prospective open, single-arm multicentre study on off-label use of tocilizumab 
in patients with severe COVID-19. Clin Exp Rheumatol 38(3):529-532

4. Zhang Y, Xiao M, Zhang S, Xia P et al (2020) Coagulopathy and antiphospholipid antibodies in patients with Covid-19. N Engl J Med 382(17):38

5. Bowles L, Platton S, Yartey N, Dave M, Lee K, Hart DP, MacDonald V, Green L, Sivapalaratnam S, Pasi KJ, MacCallum P (2020) Lupus anticoagulant and abnormal coagulation tests in patients with Covid-19. N Engl J Med. https://doi.org/10.1056/ NEJMc2013656

6. Tachil J, Tang N, Gando S et al (2020) ISTH interim guidance on recognition and management of coagulopathy of Covid 19. JTH 18:1023-1026

7. Guan WJ, Ni ZY, Hu Y, Liang WH et al (2020) Clinical characteristics of coronavirus disease 2019 in China. N Engl J Med 382:1708-1720

8. Lippi G, Favaloro E (2020) D-dimer in associated with severity of coronavirus disease 2019: a pooled analysis. Thromb Haemost. https://doi.org/10.1055/s-0044-1709650

9. Wada H, Thachil J, Di Nisio M, Mathew P, Kurosawa S, Gando S, Kim HK, Nielsen JD, Dempfle CE, Levi M, Toh CH (2013) The
Scientific Standardization Committee on DIC of the International Society on Thrombosis Haemostasis. Guidance for diagnosis and treatment of DIC from harmonization of the recommendations from three guidelines. J Thromb Haemost. https://doi.org/10.1111/ jth. 12155

10. Iba T, Levy JH, Warkentin TE, Thachil J, van der Poll T, Levi M (2019) Scientific and Standardization Committee on DIC, and the Scientific and Standardization Committee on Perioperative and Critical Care of the International Society on Thrombosis and Haemostasis. Diagnosis and management of sepsis-induced coagulopathy and disseminated intravascular coagulation. J Thromb Haemost 17(11):1989-1994

Publisher's Note Springer Nature remains neutral with regard to jurisdictional claims in published maps and institutional affiliations. 